\title{
Hepatic abscess with hepatobronchial fistula following percutaneous radiofrequency ablation for hepatocellular carcinoma: A case report
}

\author{
HUIJUAN DAI* , DAN CUI ${ }^{*}$, DAWEI LI, BO ZHAI, JIANJIAN ZHANG and JIANJUN ZHANG \\ Department of Liver Surgery, Ren Ji Hospital, School of Medicine, Shanghai Jiao Tong University, \\ Shanghai 200127, P.R. China
}

Received April 2, 2014; Accepted January 19, 2015

DOI: $10.3892 / \mathrm{ol} .2015 .3044$

\begin{abstract}
Radiofrequency ablation (RFA) has a low rate of complication and is one of the most effective and minimally invasive techniques for the treatment of liver tumors. However, a number of complications may occur in rare cases, including bronchobiliary fistula, hollow viscera perforation, diaphragmatic perforation and hernia. The present study reports a case of hepatic abscess with hepatobronchial fistula following RFA of hepatocellular carcinoma; this led to severe lung infection, respiratory failure and mortality. The present case report aims to improve understanding of the cause and mechanism of the complications arising through RFA of the liver, and highlight important factors in the prevention and management process. This case indicates that the complications of RFA may be prevented or effectively managed through preoperative evaluation, intraoperative and postoperative monitoring.
\end{abstract}

\section{Introduction}

Hepatocellular carcinoma (HCC) is the fifth most common cancer and the third highest cause of cancer-related mortality in the world $(1,2)$. Although hepatic resection or liver transplantation are the standard methods of treatment for liver cancer, thermal ablation treatment, including radiofrequency ablation (RFA), is also widely utilized. Numerous clinical trials have demonstrated that thermal ablation treatment and curative resection have similar one and three-year survival rates (95.7 and $74.4 \%$ vs. 95.1 and $78.9 \%$, respectively) for tumors $<5 \mathrm{~cm}$ in diameter $(3,4)$. Although it is a minimally

Correspondence to: Professor Jianjun Zhang, Department of Liver Surgery, Ren Ji Hospital, School of Medicine, Shanghai Jiao Tong University, 1630 Dongfang Road, Shanghai 200127, P.R. China

E-mail: daihuijuan1988@hotmail.com

\section{${ }^{*}$ Contributed equally}

Key words: hepatocellular carcinoma, radiofrequency ablation, bronchial fistula, infection invasive treatment, thermal ablation may cause a number of common clinical complications, including pain, fever, bowel injury, abdominal bleeding, bile duct injury, liver abscess and implantation metastasis of tumor cells (5). In addition, rare complications may occur post-RFA, leading to more serious consequences, such as intrahepatic infection, hemorrhage or mortality. To the best of our knowledge, no cases of hepatic abscess with hepatobronchial fistula post-RFA have been reported. The current case report describes a rare complication of an hepatic abscess, involving invasion of the lung via the diaphragm, leading to hepatobronchial fistula following hepatic RFA. Despite treatment through drainage and anti-infection medication, the patient succumbed to respiratory failure. This report aims to discuss the prevention and treatment of complications, and explore the indications and contraindications of thermal ablation. Written informed consent was obtained from the patient's family.

\section{Case report}

A 65-year-old male with a 20-year history of hepatitis B and a five-year history of liver cirrhosis was admitted to Renji Hospital (Shanghai, China) in December 2010, and subsequently diagnosed with hepatocellular carcinoma (HCC) located in segment II-III of the hepatic left lateral lobe. Five days following admission, a hepatic resection was successfully performed. The patient received regular postoperative follow-up examinations, however, one year later due to tumor recurrence, the patient was treated with three hepatic cycles of RFA. On July 31, 2012, magnetic resonance imaging during a follow-up visit revealed a tumor measuring $5.5 \times 3.5 \mathrm{~cm}$ in the right posterior hepatic lobe (Fig. 1). Due to the large size of the tumor, transarterial chemoembolization (TACE) was conducted. As this treatment did not produce a satisfactory outcome, percutaneous RFA was performed successfully under ultrasonographic guidance on August 10, 2012. Anti-inflammatory ( $0.5 \mathrm{~g}$ intravenous imipenem, every $8 \mathrm{~h}$ ), hepatoprotective $(500 \mathrm{mg}$ intravenous s-adenosylmethionine, once a day) and symptomatic treatments (electrolyte and $10 \mathrm{~g}$ albumin supplements) were also administered. The patient was discharged three days following RFA; however, after a further two days, the patient was admitted to the emergency 

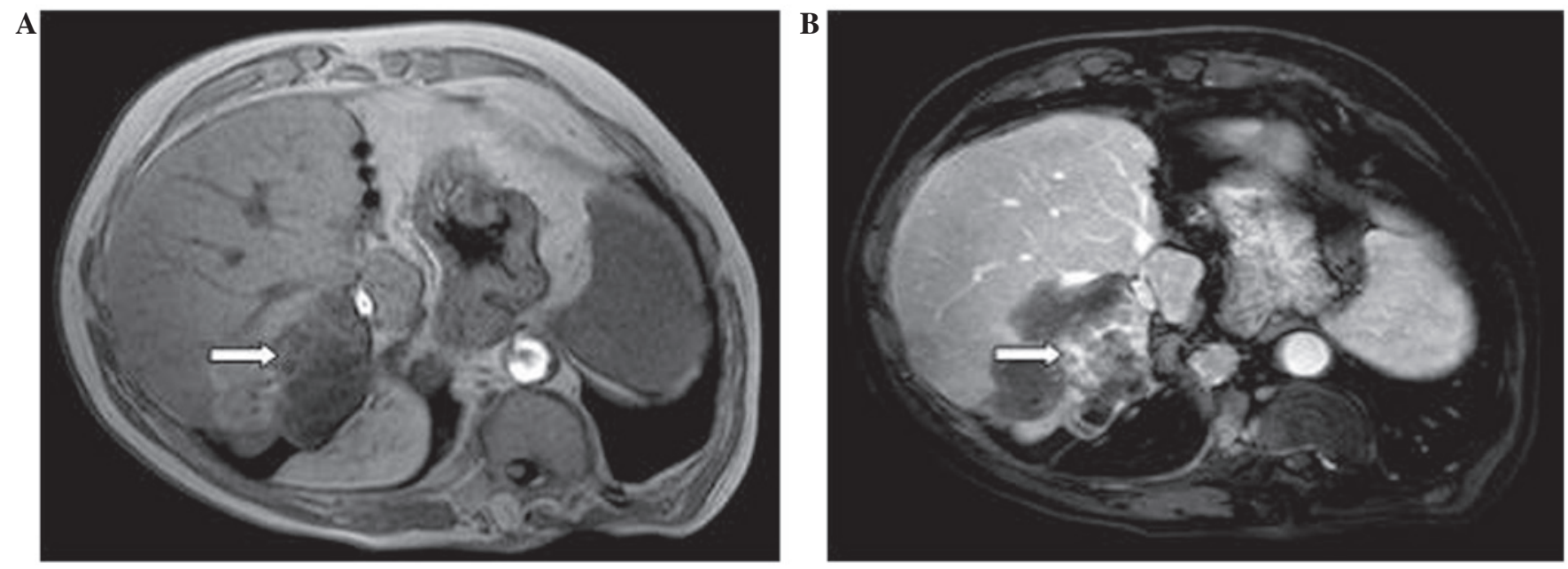

Figure 1. Magnetic resonance imaging revealed a hepatocellular carcinoma of $5.5 \times 3.5 \mathrm{~cm}$ in size in the right posterior hepatic lobe on (A) T1 phase and (B) T2 phase imaging.
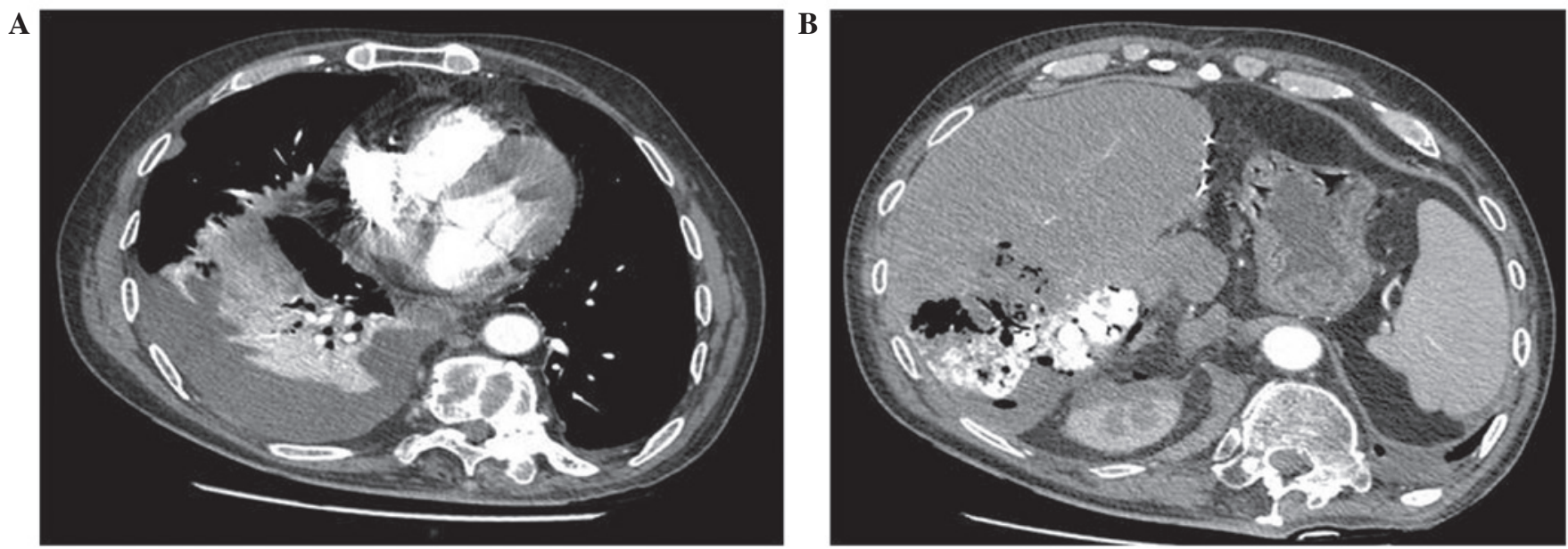

Figure 2. Computed tomography imaging following hepatic radiofrequency ablation showed (A) hepatic abscess with hepatobronchial fistula eroding through the liver capsule with perirenal fascial involvement and (B) pleural effusion with lower right pulmonary atelectasis.

room due to a sudden occurrence of hematemesis and unconsciousness, which lasted for $1 \mathrm{~h}$. Physical examination revealed that the patient's blood pressure was $90 / 60 \mathrm{mmHg}$ (normal range, 90-120/50-80 $\mathrm{mmHg}$ ) and heart rate was 160 beats/min (normal range, 60-100 beats/min); therefore hemorrhagic shock was suspected. Following hemostasis, electrolyte replacement and rehydration, and the administration of hepatoprotective and other symptomatic treatments, including parenteral nutrition, the patient stabilized with blood pressure of $110 / 70 \mathrm{mmHg}$ and a heart rate of 100 beats/min. After a further one day, the patient developed a high fever $\left(39.6^{\circ} \mathrm{C}\right)$ with chills and complained of physical weakness. Laboratory test results revealed a white blood cell count of $14.75 \times 10^{9} / 1$ (normal range, 3.70-9.20 $\times 10^{9} / 1$ ), red blood cell count of $3.48 \times 10^{12} / 1$ (normal range,3.68-5.74 $\times 10^{12} / 1$ ), platelet count of $75 \times 10^{9} / 1$ (normal range, $85-320 \times 10^{9} / 1$ ), and a hemoglobin level of $103 \mathrm{~g} / 1$ (normal range, 113-172 g/l). Biliary retrograde infection of the hepatic ablation zone was suspected due to the accumulation of gas in this region, observed on B-ultrasound examination. The patient's condition improved following the application of third-generation cephalosporin antibiotics, nutritional support, hepatoprotective treatment and other symptomatic treatments, such as rectal administration of $18.75 \mathrm{mg}$ indomethacin, once a day. On post-operative day 17, the patient experienced sudden right upper quadrant abdominal pain that radiated to the back, concurrent with dyspnea and a cough. A physical examination revealed deep abdominal tenderness without rebound pain. Computed tomography (CT) imaging of the lungs and abdomen revealed lower right pulmonary atelectasis and pleural effusion connected to the hepatic abscess (Fig. 2). Pleural fluid culture did not detect bacteria. Following treatment with anti-inflammatory drugs and thoracic cavity drainage, the patient's abdominal pain improved; however, the dyspnea was not relieved. On post-operative day 20 , the patient complained of a worsened cough accompanied by thin yellow sputum which was bitter in taste and was suspected to contain bile. Re-examination of the lungs by CT indicated that effusion was still present on the right lung field, and the hepatic abscess contained gas and had expanded, obscuring the boundary between the liver and the lung (Fig. 3). The patient exhibited shortness of breath and oxygen desaturation despite symptomatic treatments. CT imaging of the chest and abdomen indicated atelectasis in the right lower lung field, bilateral bronchiectasis in the lower 

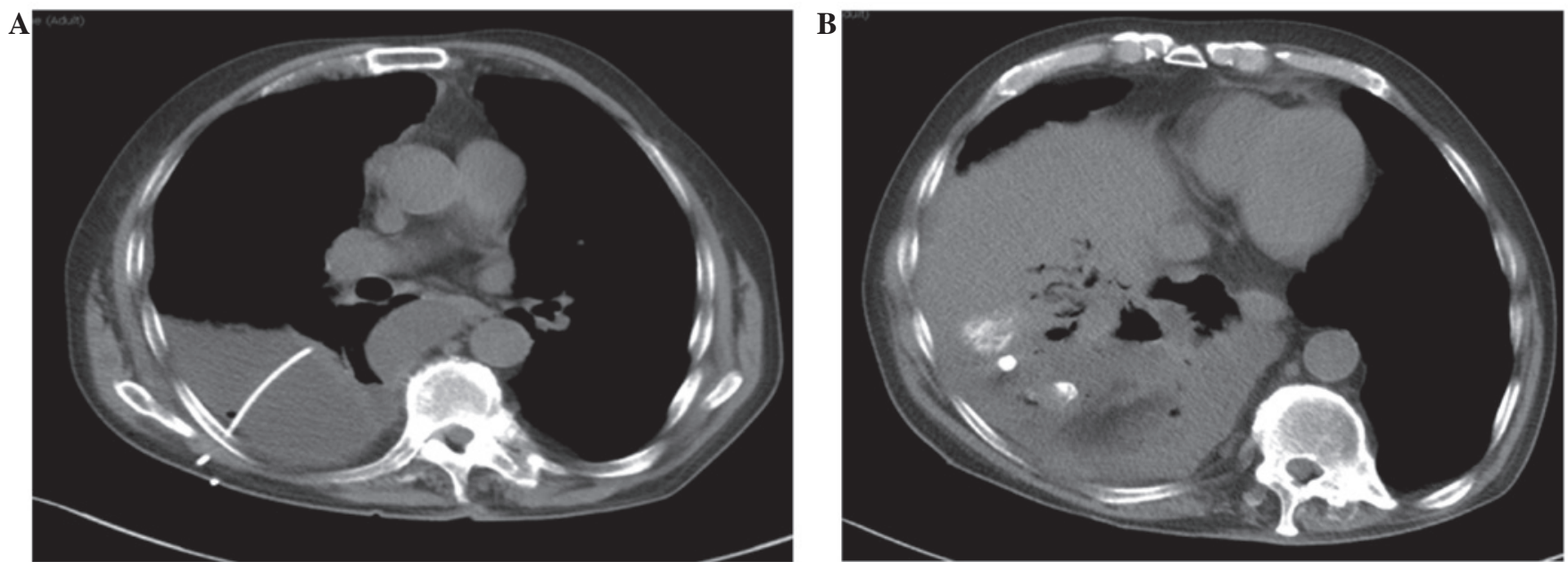

Figure 3. Computed tomography imaging showed (A) pleural effusion in the right thoracic cavity and shadow of drainage tube and (B) focus of intrahepatic ablation containing gas, which expanded to obscure the boundary between the liver and the lung.
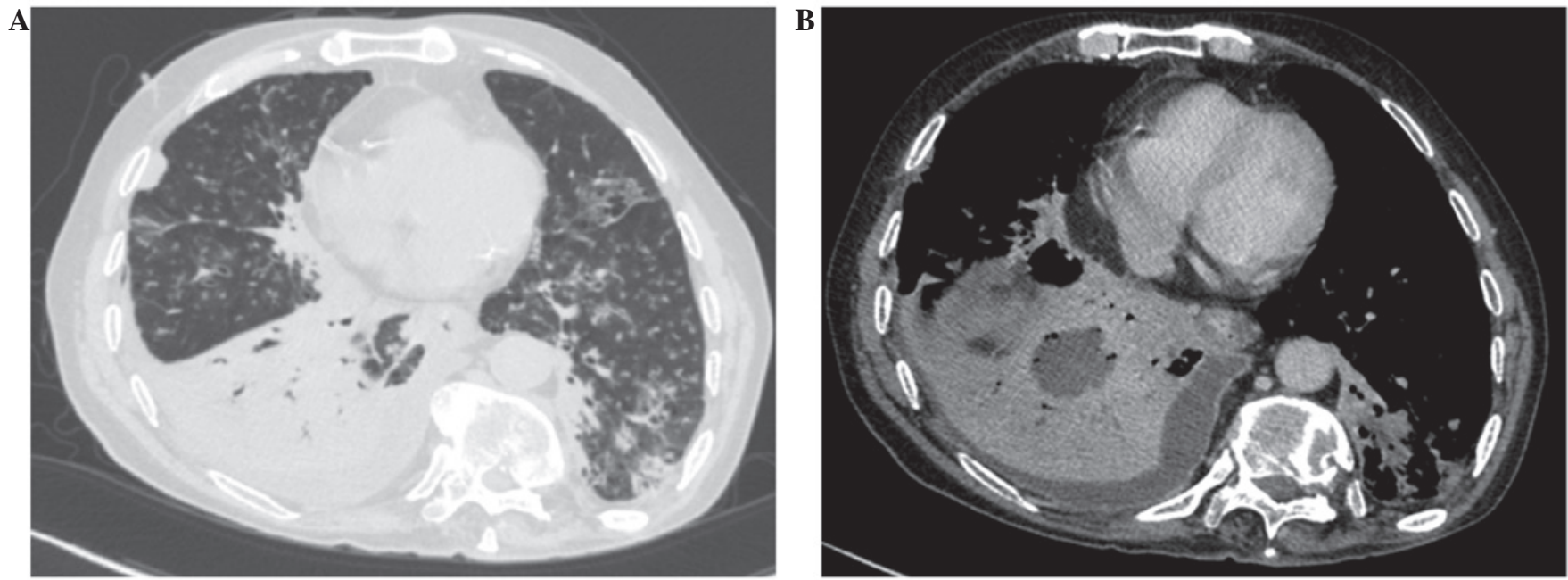

Figure 4. Computed tomography imaging showed (A) atelectasis in the right lower lung field and bilateral bronchiectasis in lower lung fields; and (B) fluidfilled space connected with intrahepatic focal ablation.

lung fields, bacterial (E. coli) lung infection and a fluid-filled space connected to the intrahepatic focal ablation (Fig. 4). The clinical symptoms and CT scan indicated a diagnosis of hepatic abscess with hepatobronchial fistula, a rare complication of RFA, which was caused by the invasion of intrahepatic infection and bile leakage one month following RFA. Despite treatment with anti-inflammatory drugs (third-generation cephalosporins) and thoracic cavity drainage, the patient succumbed to respiratory failure one day after treatment.

\section{Discussion}

Hepatocellular carcinoma, which often occurs with liver cirrhosis, is a common primary liver tumor worldwide (6). With the advancement of medical techniques, a great variety of HCC treatments have been developed. Optimal treatment must be selected according to the individual conditions. RFA is one of the most widely used local ablative therapies for small $\mathrm{HCC}$ ( $<5 \mathrm{~cm}$ in diameter), with an efficacy that is comparable to surgical resection $(7,8)$. The criteria used to determine
RFA suitability in cases of HCC include the following: A single nodule $<5 \mathrm{~cm}$ in size, or $\leq 3$ nodules each $<3 \mathrm{~cm}$ in size; Child-Pugh class A or B; and the absence of portal vein thrombosis or extrahepatic metastases (4). Compared with hepatic resection, RFA has many advantages: i) It is minimally invasive with low risk; ii) it enables excellent local tumor control; iii) it is associated with rapid recovery and promising five-year survival rates; iv) it is a multimodal approach (9). However, RFA may cause certain complications, including peritoneal hemorrhage, bile duct injury, bowel perforation, liver abscess and cancer seeding along the electrode tract, liver infarction, diaphragmatic perforation and hernia (9-12). Hepatic abscess with hepatobronchial fistula is an extremely rare complication resulting from hepatic RFA for the treatment of HCC.

In the current case, the patient's condition was poor due to multiple surgeries, and the hepatic tumor was large and tightly adjacent to the porta hepatis; this allowed necrotic tissues resulting from RFA to invade the small bile duct, which contributed to secondary infection. In the early stages of the tumor, if percutaneous drainage is unable to drain the necrotic 
tissues due to a lack of tumor tissue liquefaction, antibiotics are the only remaining strategy that may be used to control infection (13). If antibiotic therapy is ineffective, the intrahepatic infection is able to spread and may cause bacteremia (14). In the present case, the diaphragm and adjacent organs were easily invaded by the intrahepatic infectious focus close to the diaphragm. RFA damaged the bile ducts, leading to intrahepatic infection caused by leakage of bile to the hepatic abscess, and subsequently, the invasion of the diaphragm and right lung by the intrahepatic infection and bile. Due to the continual erosion by the intrahepatic infection, the lung infection could not be controlled, resulting in mortality due to the respiratory failure. Therefore, patients who have undergone RFA treatment, particularly those with large tumors, must be carefully monitored in order to prevent intrahepatic infection. Once infection is detected, anti-infective agents must be administered and drainage of the liver abscess must be performed immediately.

The present case demonstrates the importance of CT imaging of the chest, abdomen and pelvis following RFA, to detect possible thermal injuries beyond the liver. The patient's diaphragmatic defect was not formed during RFA treatment (data not shown); the hepatic abscess with hepatobronchial fistula formation primarily resulted from intrahepatic infection. The infection and bile penetrated the diaphragm causing bacterial and chemical erosion of the bronchus. In this case, medical and surgical methods were unable to eliminate the infection, which invaded the diaphragm and caused uncontrollable pulmonary infection. In such conditions, prognosis is typically poor, and the rapid prevention and/or treatment of the infection is essential. For the prevention or minimization of intraoperative and postoperative complications, the following recommendations must be considered: i) For patients whose tumor location is complicated, particularly if close to the porta hepatis, RFA must be performed under laparoscopy or by pneumoperitoneum and the appropriate RFA electrodes and frequency must be selected to avoid overheating, which may cause damage to adjacent tissues and organs (15); ii) for patients with large tumors, depending on the patient condition, preoperative TACE/TAE may be performed to reduce the tumor volume prior to RFA treatment (13); iii) during and following the surgery, surgeons must carefully monitor the patient's vital signs.

The current case report describes a case of hepatic abscess with hepatobronchial fistula, occurring five days following hepatic RFA. To the best of our knowledge, no other reports of this condition have been published. The unfavorable outcome of the present case demonstrates that, although RFA is a minimally invasive and effective method to treat liver cancer, the indications of RFA, such as early tumor stage and small tumor size, must be precisely determined, and careful monitoring for potential postoperative complications that may lead to mortality must be conducted. This is essential to improve the success rate of RFA.

\section{Acknowledgements}

This study was supported by a grant from sTNFr-13 horizontal subject (no. 101005.335 ) to Jianjun Zhang.

\section{References}

1. Lau WY, Leung TW, Yu SC, and Ho SK: Percutaneous local ablative therapy for hepatocellular carcinoma: a review and look into the future. Ann Surg 237: 171-179, 2003.

2. Livraghi T, Mäkisalo H and Line PD: Treatment options in hepatocellular carcinoma today. Scand J Surg 100: 22-29, 2011.

3. Tombesi P, Di Vece F and Sartori S: Resection vs thermal ablation of small hepatocellular carcinoma: What's the first choice? World J Radiol 5: 1-4, 2013.

4. Baldan A, Marino D, DE Giorgio M, et al; Gene - Gruppo Epatocarcinoma NORD-EST: Percutaneous radiofrequency thermal ablation for hepatocellular carcinoma. Aliment Pharmacol Ther 24: 1495-1501, 2006.

5. Howenstein MJ and Sato KT: Complications of radiofrequency ablation of hepatic, pulmonary, and renal neoplasms. Semin Intervent Radiol 27: 285-295, 2010.

6. Shirai K, Tamai H, Shingaki N, et al: Clinical features and risk factors of extrahepatic seeding after percutaneous radiofrequency ablation for hepatocellular carcinoma. Hepatol Res 41: 738-745, 2011.

7. Khan MR, Poon RT, $\mathrm{Ng} \mathrm{KK}$, et al: Comparison of percutaneous and surgical approaches for radiofrequency ablation of small and medium hepatocellular carcinoma. Arch Surg 142: 1136-1143, 2007.

8. Guglielmi A, Ruzzenente A, Valdegamberi A, Pachera S, et al: Radiofrequency ablation versus surgical resection for the treatment of hepatocellular carcinoma in cirrhosis. J Gastrointest Surg 12: 192-198, 2008.

9. Rhim H and Lim HK: Radiofrequency ablation of hepatocellular carcinoma: pros and cons. Gut Liver 4: S113-S118, 2010.

10. Chiu YC, Chuang CH, Tsai HM and Chen CY: Massive hepatic infarction after pure ethanol injection and radiofrequency ablation therapy for hepatocellular carcinoma: a case report. Kaohsiung J Med Sci 25: 156-159, 2009.

11. Zhou M, He H, Cai H, et al: Diaphragmatic perforation with colonic herniation due to hepatic radiofrequency ablation: A case report and review of the literature. Oncol Lett 6: 1719-1722, 2013.

12. Ke S, Ding XM, Qian XJ, et al: Radiofrequency ablation of hepatocellular carcinoma sized $>3$ and $\leq 5 \mathrm{~cm}$ : is ablative margin of more than $1 \mathrm{~cm}$ justified? World J Gastroenterol 19: 7389-7398, 2013

13. Zhang Z, Zhuang Z, Xu Z, et al: Post-operative pericardial effusion following treatment of small hepatocellular carcinoma with radiofrequency ablation: A case report. Oncol Lett 7: 345-348, 2014

14. Thiemann M, Benhidjeb T, Anders S, et al: Hepato-pericardial fistula following radiofrequency ablation (RFA) for liver metastasis: a case report and review of the literature.Langenbecks Arch Surg 393: 1013-1016, 2008

15. Wood TF, Rose DM, Chung M, et al: Radiofrequency ablation of 231 unresectable hepatic tumors: indications, limitations, and complications. Ann Surg Oncol 7: 593-600, 2000. 